\title{
Local anisotropy and giant enhancement of local electromagnetic fields in fractal aggregates of metal nanoparticles
}

\author{
Sergei V. Karpov \\ L.V. Kirensky Institute of Physics, Russian Academy of Sciences, Siberian Branch, Krasnoyarsk 660036, Russia \\ Valeriy S. Gerasimov and Ivan L. Isaev \\ Department of Physics and Engineering, Krasnoyarsk State Technical University, Krasnoyarsk 660028, Russia \\ Vadim A. Markel \\ Departments of Radiology and Bioengineering, University of Pennsylvania, Philadelphia, PA 19104
}

(Dated: November 21, 2018)

\begin{abstract}
We have shown within the quasistatic approximation that the giant fluctuations of local electromagnetic field in random fractal aggregates of silver nanospheres are strongly correlated with a local anisotropy factor $S$ which is defined in this paper. The latter is a purely geometrical parameter which characterizes the deviation of local environment of a given nanosphere in an aggregate from spherical symmetry. Therefore, it is possible to predict the sites with anomalously large local fields in an aggregate without explicitly solving the electromagnetic problem. We have also demonstrated that the average (over nanospheres) value of $S$ does not depend noticeably on the fractal dimension $D$, except when $D$ approaches the trivial limit $D=3$. In this case, as one can expect, the average local environment becomes spherically symmetrical and $S$ approaches zero. This corresponds to the well-known fact that in trivial aggregates fluctuations of local electromagnetic fields are much weaker than in fractal aggregates. Thus, we find that, within the quasistatics, the large-scale geometry does not have a significant impact on local electromagnetic responses in nanoaggregates in a wide range of fractal dimensions. However, this prediction is expected to be not correct in aggregates which are sufficiently large for the intermediate- and radiation-zone interaction of individual nanospheres to become important.
\end{abstract}

\section{INTRODUCTION}

Electromagnetic properties of fractal nanostructures have continuously attracted attention since the late 1980ies due to their rather unusual physical properties and the possibility of numerous applications, as described in several reviews of the subject $1,1,2,3,4,5,6]$. Of special interest are aggregates of metal nanoparticles in hydrosols and percolation clusters (metal-dielectric composites) which have, in particular, exhibited the effects of giant enhancement of nonlinear-optical responses 7, 8, 9, 10], inhomogeneous localization of electromagnetic eigenmodes [11, 12], and optical memory [13, 14, 15, 16, 17].

Theoretical description of the electromagnetic responses of disordered fractal aggregates has been closely interconnected with numerical simulations. This is due to the fact that a fully analytic solution to the problem of interaction of an electromagnetic field with a large random fractal aggregate has not been devised. Some approximate theoretical approaches were based on the first Born 18] and mean-field [19] approximations, approximations based on few-body interaction (binary 20, 21. or binary-ternary 12 approximations), and various phenomenological scaling laws [8, 20, 21, 22, 23, 24]. The first Born and the mean-field approximations are not, generally, useful in the spectral regions where excitation is resonant. While off-resonant electromagnetic properties of fractal aggregates are of interest in atmospheric physics 25, 26, 27], the research in electromagnetics of metal fractal aggregates is primarily focused on resonant interactions. The few-body approximations and the scaling laws proved to be very useful for qualitative theoretical description at the early stages of research. However, increasingly more realistic simulations revealed that these approaches do not provide quantitative results. Currently, they are effectively obsolete. A brief overview of the progression of numerical models used to simulate electromagnetic responses of fractal aggregates is given in the next paragraph.

The theoretical and computational description has been primarily based on a model of an aggregate of $N$ touching identical spherical nanoparticles. Each nanoparticle, and sometimes the aggregate as a whole, are assumed to be much smaller in size than the external wavelength. (Polydisperse aggregates built from spheres of different size have also been recently addressed 28, 29, 30].) In order for an aggregate to be considered fractal, the number of primary spheres, must be large, typically, $\gtrsim 10^{3}$. Initially, simulations were based on the dipole approximation. In this approximation, each sphere is assigned a dipole moment located at its center. The spheres then interact with each other and the external field via dipole radiation fields as described by $3 N$ coupled-dipole equations 21]. In the late 1980-ies and early 1990-ies, numerical solution of dense linear systems of $\gtrsim 10^{3}$ equations was a difficult computational task. Therefore a model of diluted aggregates was adopted and used, for example, in Refs. 8, 21, 22, 31, 32, 33, 34]. According to this model, an aggregate of $N$ touching spheres (where $N$ can be very large) is diluted, i.e., 
spheres were randomly removed from the aggregate with the probability $1-p$, where $p \ll 1$. Then the coordinates of the remaining spheres are rescaled according to $\mathbf{r} \rightarrow p^{1 / D} \mathbf{r}$, where $D$ is the fractal dimension. This procedure does not change the density-density correlation function of the aggregate in some intermediate region. However, it does change the local structure of the aggregate substantially. The few-body approximations and scaling laws were largely validated with the model of diluted aggregates. However, when computations with non-diluted clusters became feasible, it was found that both the few-body approximations and the scaling laws are inaccurate 35. The deviation from the scaling laws has been explained by the phenomenon of inhomogeneous localization [3]; however, the theoretical relation of this phenomena to the aggregate geometry has not been clarified. Additionally, it has been well known that account of excitation of higher multipole modes is important for touching nanoparticles, even when the size of each nanoparticle is much smaller than the external wavelength [36, 37, 38, 39]. In particular, the dipole approximation failed to properly describe experimentally observed red shifts in extinction spectra of colloid aggregates [35, 40]. To remediate this problem, a phenomenological model of geometrical renormalization have been introduced [35, 41] and, recently, computations beyond the dipole approximation have been performed [42].

The combination of findings contained in the abovecited references strongly suggest that the local structure of aggregates is of primary importance. However, the local structure of random fractal nanoaggregates has not been so far the focus of research. In this paper we, for the first time, point to a strong correlation between the anisotropy of local environment and enhancement of local field in fractal aggregates within the quasistatic approximation. In particular, we find that the correlation coefficient of the local anisotropy factor $S$ (introduced below) and the value of a local squared dipole moment $|\mathbf{d}|^{2}$ can be as high as 0.75 and tends to grow with the wavelength. We have found that the average local anisotropy factor is almost independent of fractal dimension in the range $1.7<D<2.8$. Note that this result is expected to change in large aggregates where intermediate- and far-zone interaction is important.

The paper is organized as follows. In Section [11 the local anisotropy factor is introduced. The dependence of the local anisotropy factor on the fractal dimension of aggregates and other parameters for computer-simulated fractals is discussed in Section III] Section IV contains results concerning the correlation of local electromagnetic fields and the local anisotropy factor. The electromagnetic calculations in this section were performed with the method of coupled multipoles 39, 42, e.g., without the dipole approximation. Finally, Section $\square$ contains a summary of obtained results.

\section{DEFINITION OF THE LOCAL ANISOTROPY FACTOR}

The definition of local anisotropy factor introduced in this paper is based on an analogy with ellipsoids. An ellipsoid is a geometrical object that can exhibit either perfect spherical symmetry, or strong anisotropy, depending on its eccentricity.

Consider a general ellipsoid excited by a linearly polarized monochromatic external wave of amplitude $\mathbf{E}_{0}$. In the quasistatic limit, the polarization $\mathbf{P}$ inside the ellipsoid is independent of position and can be found from

$$
4 \pi\left[\frac{1}{\epsilon-1}+\left(\frac{1}{3}-\hat{Q}\right)\right] \mathbf{P}=\mathbf{E}_{0}
$$

where the tensor $\hat{Q}$ is given by

$$
\hat{Q}=\int_{V} \hat{G}_{0}\left(0, \mathbf{r}^{\prime}\right) d^{3} r^{\prime}
$$

Here $\hat{G}_{0}\left(\mathbf{r}, \mathbf{r}^{\prime}\right)$ is the regular part of the quasistatic freespace dyadic Green's function for the electric field. The integral is taken over the volume of the ellipsoid, $V$, and is independent of position. Therefore, it is evaluated at the center of ellipsoid, $\mathbf{r}=0$. A unique property of ellipsoids is that $\hat{Q}$ is diagonal in the reference frame whose axes are collinear to the main axes of the ellipsoid. Correspondingly, if $E_{0, \alpha}$ are the Cartesian components of the external electric field in the same reference frame, the solution to (1) is

$$
P_{\alpha}=\frac{E_{0, \alpha}}{4 \pi\left[1 /(\epsilon-1)+\nu_{\alpha}\right]},
$$

where $\nu_{\alpha}$ are the depolarization factors related to the principal values of $\hat{Q}$ by

$$
\nu_{\alpha}=1 / 3-Q_{\alpha}
$$

In the case of spherical symmetry $(e=0), Q_{\alpha}=0$ and $\nu_{\alpha}=1 / 3$. For an ellipsoid of nonzero eccentricity, the depolarization factors become different from $1 / 3$. Thus, for example, if $e=1$, we have $\nu_{1}=\nu_{2}=0, \nu_{3}=1$ for an oblate ellipsoid (infinitely thin circular disk) or $\nu_{1}=\nu_{2}=1 / 2, \nu_{3}=0$ for a prolate ellipsoid (infinitely thin needle). The anisotropy factor $S$ can be defined as dispersion of the depolarization factors:

$$
S^{2}=\left\langle\nu_{\alpha}^{2}\right\rangle-\left\langle\nu_{\alpha}\right\rangle^{2}
$$

Obviously, this parameter is zero for a sphere and positive for any ellipsoid of nonzero eccentricity. In particular, for the infinitely thin needle, $S=1 / 3 \sqrt{2}$ and for an infinitely thin circular disk, $S=\sqrt{2} / 3$. The latter is the maximum possible value for $S$ given the constraint $\sum_{\alpha} \nu_{\alpha}=1$. 
Now we extend the definition of the depolarization tensor to include particles of arbitrary shape. Namely, for an arbitrary system occupying some volume $V$, we define

$$
\hat{\nu}(\mathbf{r})=\frac{1}{3} \hat{I}-\int_{V} \hat{G}_{0}\left(\mathbf{r}, \mathbf{r}^{\prime}\right) d^{3} r^{\prime}
$$

where $\hat{I}$ is the unity tensor. If $V$ is of general shape, the result of integration in the right-hand side of (6) is position-dependent. Therefore, the tensor $\hat{\nu}(\mathbf{r})$ depends on the point $\mathbf{r}$ it is evaluated at and is referred to here as local. Similarly to the case of ellipsoids, this tensor can be diagonalized. Then we can use the principal values $\nu_{\alpha}(\mathbf{r})$ to calculate the anisotropy factor according to (5).

In this paper we consider aggregates of (possibly, polydisperse) spheres whose centers are located at points $\mathbf{r}_{i}$ and radii are denoted by $a_{i}$. In this case, the expression for $\hat{\nu}(\mathbf{r})$ is simplified. We use

$$
\int_{\left|\mathbf{r}^{\prime}-\mathbf{r}_{i}\right|<a_{i}} \hat{\mathbf{G}}_{0}\left(\mathbf{r}, \mathbf{r}^{\prime}\right) d^{3} r^{\prime}= \begin{cases}v_{i} \hat{\mathbf{G}}_{0}\left(\mathbf{r}, \mathbf{r}_{i}\right), & \text { if }\left|\mathbf{r}-\mathbf{r}_{i}\right|>a_{i} \\ 0, & \text { if }\left|\mathbf{r}-\mathbf{r}_{i}\right|<a_{i}\end{cases}
$$

to obtain

$$
\hat{\nu}_{i} \equiv \hat{\nu}\left(\mathbf{r}_{i}\right)=\frac{1}{3} \hat{I}-\sum_{j \neq i} v_{j} \hat{G}_{0}\left(\mathbf{r}_{i}, \mathbf{r}_{j}\right)
$$

Here $v_{i}=4 \pi a_{i}^{3} / 3$ is the volume of the $i$-th sphere and the components of $\hat{G}_{0}\left(\mathbf{r}_{i}, \mathbf{r}_{j}\right)$ are given by

$$
\left(G_{0}\left(\mathbf{r}_{i}, \mathbf{r}_{j}\right)\right)_{\alpha \beta}=\frac{\delta_{\alpha \beta}-3 n_{\alpha}^{(i j)} n_{\beta}^{(i j)}}{r_{i j}^{3}}
$$

where $\mathbf{r}_{i j}=\mathbf{r}_{i}-\mathbf{r}_{j}$ and $\mathbf{n}^{(i j)}=\mathbf{r}_{i j} / r_{i j}$.

Diagonalization of the tensor $\hat{\nu}_{i}$ and calculation of the dispersion of its principal values gives the local anisotropy factor $S_{i}$. This parameter quantifies the degree of anisotropy of the local environment of the $i$-th sphere.

A few notes about the introduced definition must be made. First, the principal value $\nu_{\alpha}$ obtained as described above are purely geometrical characteristics of an object. They are related to the Bergman-Milton spectral parameters 43] only in the special case of ellipsoidal (more generally, spheroidal) shape of $V$. Obtaining the BergmanMilton spectral parameters requires diagonalization of the integral operator $W$ with the kernel $G_{0}\left(\mathbf{r}, \mathbf{r}^{\prime}\right), \mathbf{r}, \mathbf{r}^{\prime} \in$ $V$. This is a much more complicated task than diagonalization of the tensor $\hat{Q}(\mathbf{r})=\int_{V} G_{0}\left(\mathbf{r}, \mathbf{r}^{\prime}\right) d^{3} r^{\prime}$ at a given point $\mathbf{r}$. In particular, $\hat{Q}(\mathbf{r})$ is three-dimensional, while $W$ is infinite-dimensional. Correspondingly, the number of Bergman-Milton parameters is infinite (although only three of them have non-zero oscillator strengths in the case of spheroids), while the tensor $\hat{\nu}(\mathbf{r})$ has only three principal values. Second, the principal values $\nu_{\alpha}(\mathbf{r})$ are not constrained, in general, by the conditions $0 \leq \nu_{\alpha} \leq 1$ and $\sum_{\alpha} \nu_{\alpha}=1$. This also distinguishes them from the Bergman-Milton spectral parameters. Next, the parameter $S_{i}$ depends on the coordinates of all nanoparticles in the aggregate with $j \neq i$. However, due to the fast cubic decay of the near-field component of the dipole radiation field, the neighbors within few coordinate spheres of the $i$-th site give the largest input to $S_{i}$. This justifies the locality of $S_{i}$, as it only weakly depends on the large scale structure. This statement needs to be qualified in aggregates large enough so that interaction in the far-zone becomes important. Even without account of retardation, the locality of $S_{i}$ can be violated in aggregates with the fractal dimension close to 3 (or in random non-fractal composites), due the logarithmic divergence of the integral $\int r^{-3} d^{3} r$ at infinity. We do not expect these effects to be important in most aggregates of practical interest with the fractal dimension in the range $D<2.7$ and do not consider them in this paper.

Finally, the introduced parameter is not sensitive to the wavelength and electromagnetic properties of the scattering material. Therefore, we do not expect it to be a good indicator of local electromagnetic response at all wavelengths. It is also independent of the incident polarization. A possible definition of a polarization-sensitive anisotropy factor is

$$
S_{i}\left(\mathbf{E}_{0}\right)=\frac{\mathbf{E}_{0}^{*} \cdot \hat{\nu}_{i} \mathbf{E}_{\mathbf{0}}}{\left|\mathbf{E}_{0}\right|^{2}} .
$$

Another possible definition is

$$
S_{i}^{2}\left(\mathbf{E}_{0}\right)=\frac{\left|\hat{\nu}_{i} \mathbf{E}_{\mathbf{0}}\right|^{2}}{\left|\mathbf{E}_{0}\right|^{2}}
$$

Note that the definitions (10), (11) are not used in this paper.

\section{RESULTS: SIMULATIONS OF GEOMETRICAL PROPERTIES}

Since the unique electromagnetic properties of colloid aggregates are often attributed to their fractal structure, we have studied computer-generated aggregates with various fractal dimensions. We have generated quasi-random off-lattice aggregates with varying fractal dimension $D$ using the algorithm described in 42 . This algorithm simulates the stochastic dynamics of individual nanoparticles and sub-aggregates in a solution with the account of random (Brownian) forces, as well as deterministic interparticle (the Van-der-Waals and Coulomb) and external potentials. Discrete Newtonian mechanics was implemented with a sufficiently small time step, such that the spatial translation of any particle (sphere) at each step is much smaller than its diameter. Rotation of 
aggregates was taken into account. We have used both monodisperse $\left(a_{i}=\right.$ const $)$ and polydisperse nanospheres ( $a_{i}$ were randomly distributed according to the Poisson distribution). The fractal dimension of obtained aggregates was tuned in the interval $1.7<D<3.0$ by varying the initial density of spheres prior to the aggregation process. The numerical value of $D$ was calculated from the linear regression of the pair density-density correlation function which, in the intermediate asymptote region, has the scaling form $g(r) \propto r^{D-3}$.

The aggregation was simulated in a cubic volume with elastically reflecting boundaries. In the limit of low initial concentration of particles and the size of the cubic cell of $\sim 200 a$ or more, the obtained aggregates have the typical fractal dimension $D \approx 1.7$. When the initial concentration increases, $D$ approaches the trivial limit $D=3$. As a graphical illustration of generated fractals, we show in Fig. 1 a large aggregate and values of the local anisotropy factor $S$ at some selected sites.

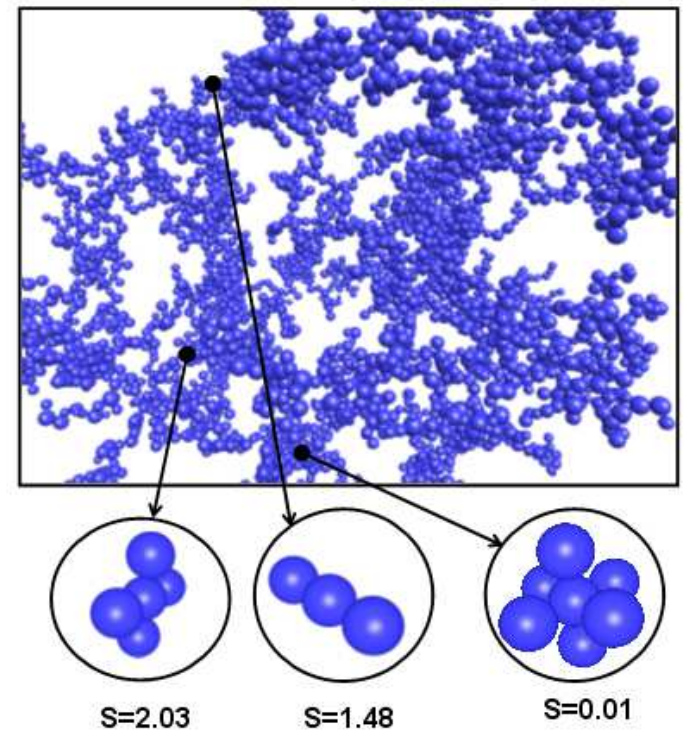

Fig. 1. Local anisotropy factor $S$ for selected sites in a large aggregate with $N=5000$ and $D \approx 1.8$.

We start with a discussion of results for monodisperse aggregates, i.e., for aggregates built of identical spheres. In Fig. 2, we illustrate the dependence of the average (over individual particles in an aggregate) value of the $S$ on fractal dimension $D$. Aggregates with $1.70<D<2.25$ are characterized by moderate average values of local anisotropy factor, almost independently of $D$. We can argue that such aggregates differ only on large scale but have similar local structure. In other words, the local environment of each particle is, on average, the same, independently of $D$. As $D$ approaches the critical value $D=3$, the local anisotropy factor quickly drops. This corresponds to the fact that trivial (non-fractal) aggregates are characterized by almost isotropic local environment and relatively weak fluctuations of density. We have also calculated the average $S$ for two types of lattice aggregates traditionally used in electrodynamic calculations. The results are shown by centered symbols in Fig. 2.

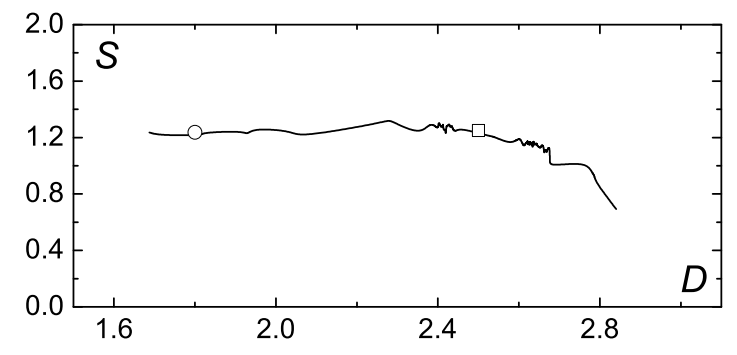

Fig. 2. Average local anisotropy factor $S$ vs fractal dimension $D$ for 121 random aggregates with $N=800$ in each. Numerical value of $D$ was computed separately for each aggregate from linear regression of the density-density correlation function in the intermediate asymptote region. The open circle shows the average values of $S$ for lattice Meakin aggregates [44] $(D \approx 1.8)$ and the open square shows the same value for a set of Witten-Sander aggregates 45] $(D \approx 2.5)$.

Real colloid aggregates are strongly polydisperse. Typically, they contain particles of sizes ranging from $5 \mathrm{~nm}$ to $30 \mathrm{~nm}$ [1, 46]. We have investigated the dependence $S(D)$ for several ensembles of polydisperse aggregates with different ratios of the maximum and minimum sphere radiuses, $a_{\max }$ and $a_{\min }$. We have used a discrete Poisson distribution of particle sizes with the number of samples equal to 11 [the discrete step in particle size was $\left.\Delta a=\left(a_{\max }-a_{\min }\right) / 10\right]$. The dependence of local anisotropy factor on the fractal dimensions $D$ is shown in Fig. 3. Note that no signifact effect doe to the polidispersity was found.

It is interesting to note that the average local anisotropy factor does not depend on the distance of a given site from the center of mass of the aggregate. This is illustrated in Fig. 4. Here we plot the value of $S$ averaged over all nanospheres within a spherical shell drawn arond the center of mass of the aggregate as a function of the shell radius (see figure captions for more detail).

In Fig. 5, we also plot the fraction of particles in an aggregate with local anisotropy factor exceeding $60 \%$ of the maximum value for that aggregate as a function of fractal dimension. It can be seen that in typical aggregates with fractal dimensions of practical interest, only small fraction of particles is placed in highly anisotropic environment. In Fig. 6, an example of an aggregate is 


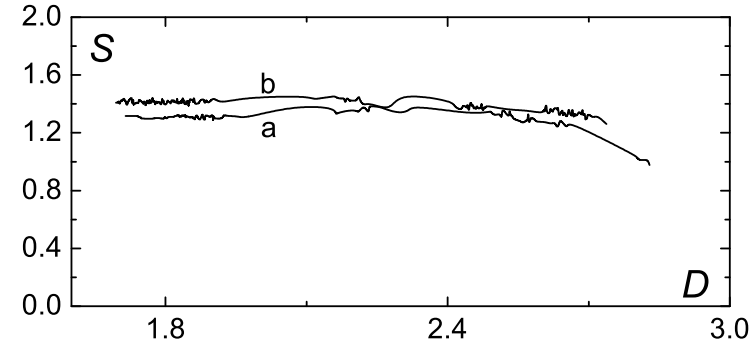

Fig. 3. Local anisotropy factor $S$ factor vs fractal dimensions $D$ for polydisperse aggregates with $N=800$ and Poisson particle size distribution. The ratio of the maximum and minimum particle radii is $a_{\max } / a_{\min }=2$ (153 random aggregates) for curve (a) and $a_{\max } / a_{\min }=3$ (297 random aggregates) for curve (b). Numerical value of $D$ was computed separately for each aggregate from linear regression of the density-density correlation function in the intermediate asymptote region.

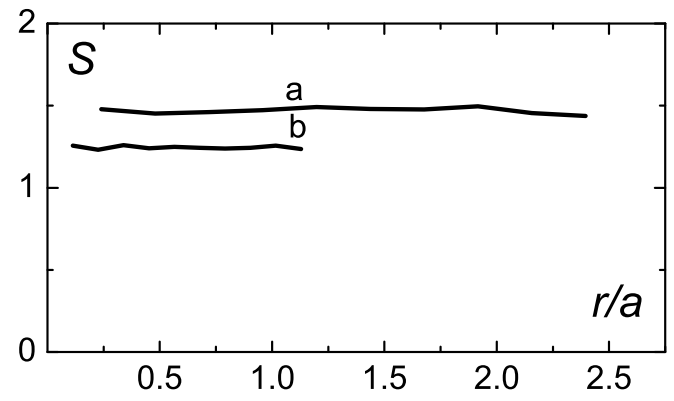

Fig. 4. Local anisotropy factor $S$ vs the relative distance to the center of mass of an aggregate, $r / a$. The hystogram is built with the step $R_{g} / 10$, where $R_{g}$ is the gyration radius of the aggregate, and $S$ was averaged over all particles located within 10 spherical shells drawn around the aggregate's center of mass for $N=10,000$ (a) and $N=3,000$ (b).

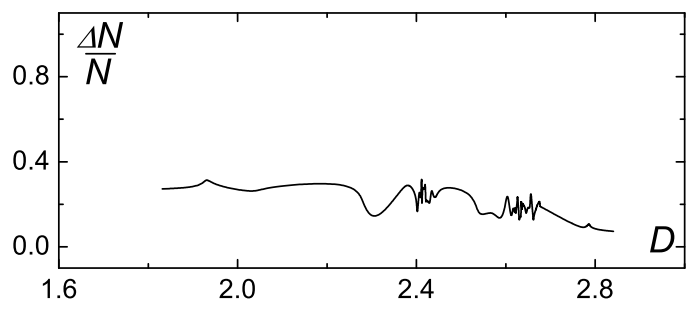

Fig. 5. Dependence of the fraction $\Delta N / N$ of sites in an monodisperse aggregate with the value of local anisotropy factor exceeding $60 \%$ of its maximum value for the same aggregate; $N=800$.

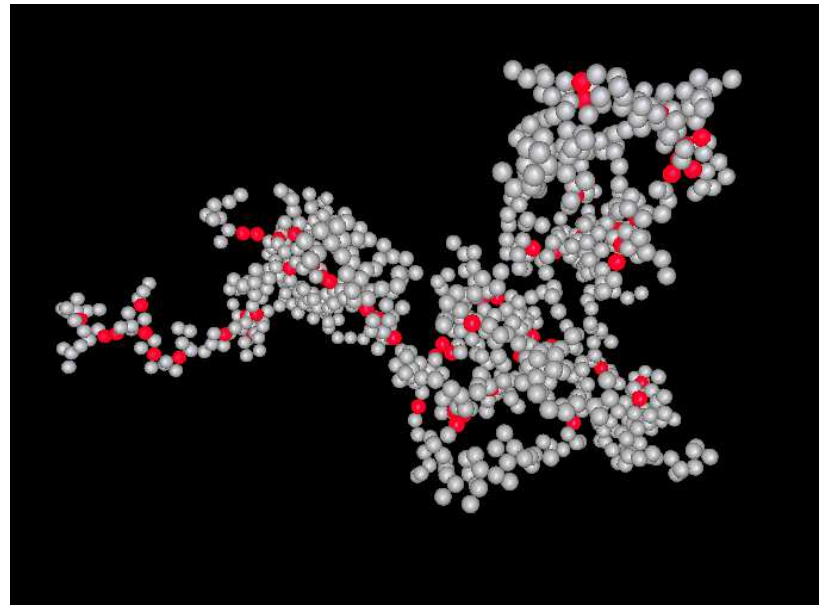

Fig. 6. Sites in a fractal aggregate $(D \approx 1.8, N=800)$ with relative values of the local anisotropy factor exceeding $80 \%$ of the maximum value for the same aggregate, $S_{\max }=2.29$.
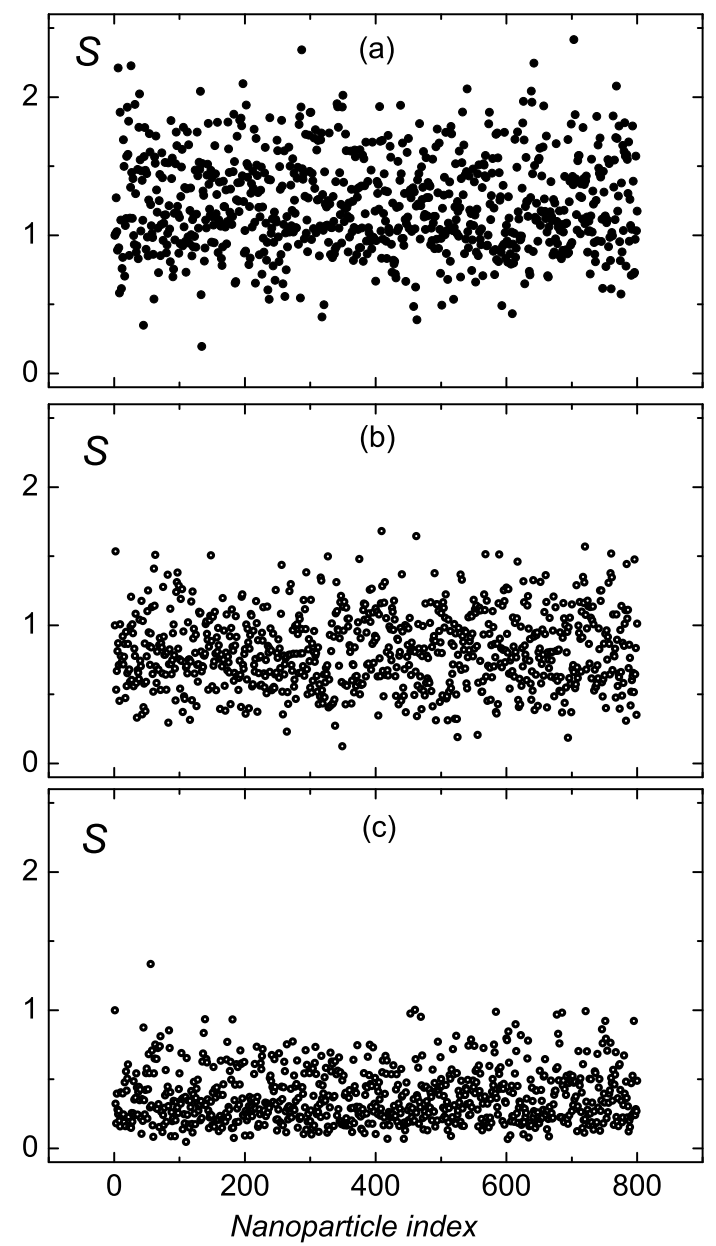

Fig. 7. Local anisotropy factor $S$ for different particles in a fractal aggregate with $N=800$ and $D \approx 1.8$ (a) compared to those in random gas of identical hard spheres.Average distance between centers of two nearest neighbor spheres $R_{\mathrm{nn}} / a=2.12$ (b) and $R_{\mathrm{nn}} / a=2.90$ (c). 
shown with the sites of relatively high local anisotropy emphasized by different color (shade of grey).

Finally, we compare the local anisotropy factors for all particles of monodisperse fractal aggregate and non-fractal random gas of hard spheres $(N=800$ in both cases). In Fig. 7a we plot these quantities for a fractal aggregate with $D \approx 1.8$. All local anisotropy factors $S_{i}$ are shown for $i=1, \ldots, 800$. In Fig. 7b,c, the same quantities are plotted for a random gas of identical hard spheres of radius $a$ distributed in a volume with the density corresponding to the average distance between the centers of nearest neighbor spheres equal to $R_{\mathrm{nn}}$; value of the ratio $R_{\mathrm{nn}} / a$ are indicated in the figure caption. It can be seen that the fractal aggregate contains sites with much higher values of local anisotropy factor than random gas. As one could expect, the local anisotropy factors become smaller when the density of random gas decreases. However, a fractal aggregate, although has zero asymptotic density in the limit $N \rightarrow \infty$, always retains approximately constant fraction of sites with relatively high local anisotropy.

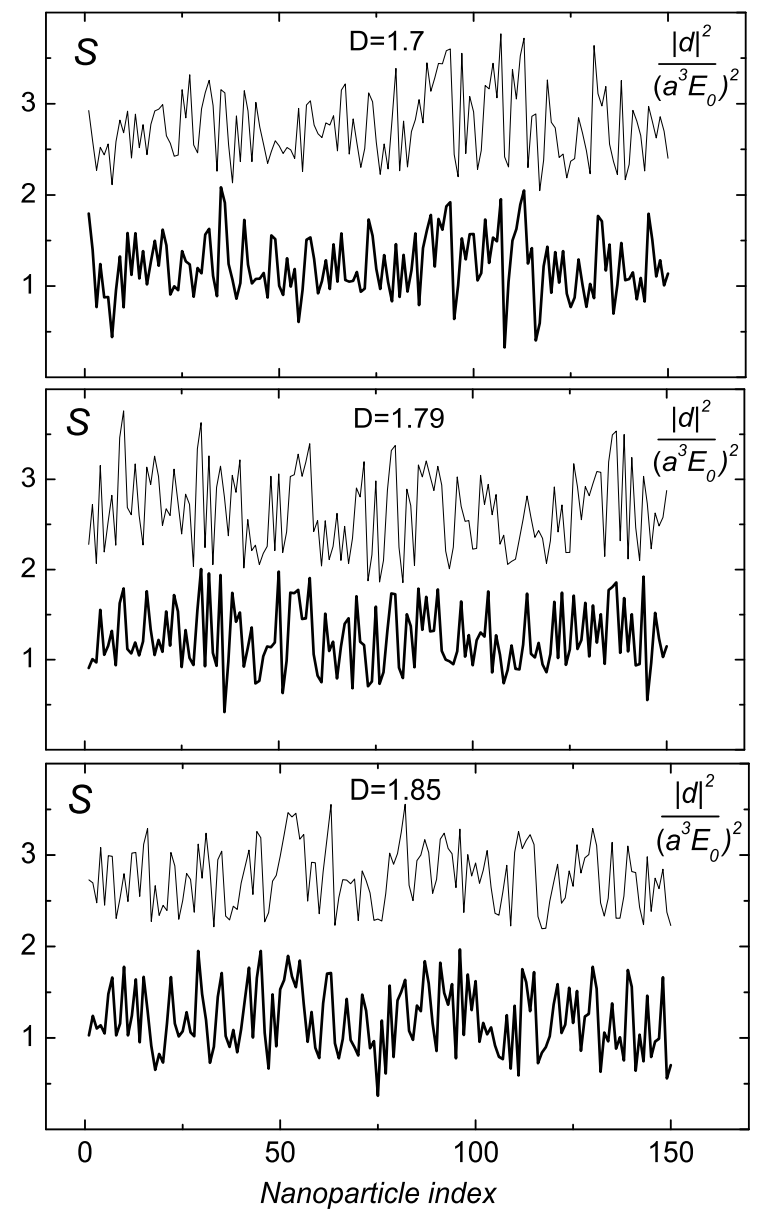

Fig. 8. Local anisotropy factor $\left(S_{i}\right)$ (thick line) and local dipole moments squared $\left|\mathbf{d}_{i}\right|^{2}$ (thin line) for different particles in a monodisperse aggregate with $N=150$ and fractal dimension $D=1.70, D=1.79$, and $D=1.85$, computed at the wavelength $\lambda=703 \mathrm{~nm}$.

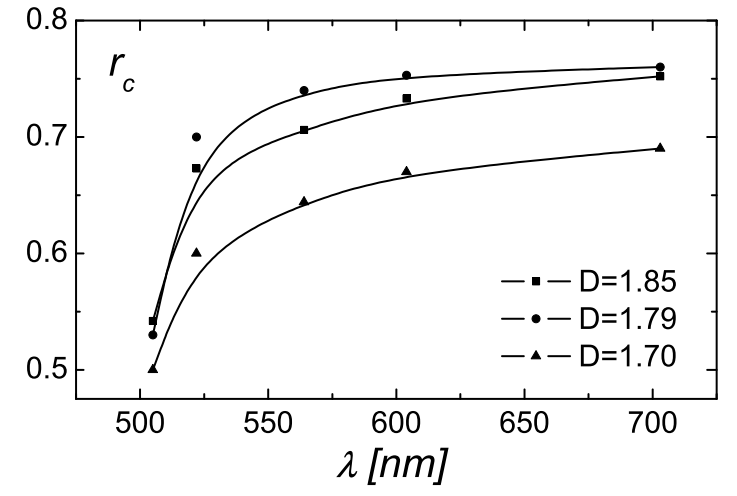

Fig. 9. Correlation between $S_{i}$ and $\left|\mathbf{d}_{i}\right|^{2}$ as a function of wavelength for monodisperse aggregates with $N=150$ and fractal dimension $D=1.70, D=1.79$, and $D=1.85$.

\section{COMPARISON OF STRUCTURAL AND ELECTRODYNAMIC PROPERTIES OF FRACTAL NANOAGGREGATES}

The main idea of this paper is that there is certain correlation between local structure and local electromagnetic fields in fractal nanoaggregates. This assumption is confirmed by numerical simulations presented in this Section.

We have computed optical responses of aggregates of nanospheres using the method of coupled multipoles 39, 42]. Calculations were performed for monodisperse aggregates built of $N=150$ silver nanospheres of constant radius $a=5 \mathrm{~nm}$ and placed in vacuum. To facilitate convergence with the maximum order of multipoles included, we have introduced a surface layer of thickness $h=0.05 a$. The dielectric constant of the layer was chosen to be the same as that of the vacuum, $\epsilon=1$. We have used experimental values of the optical constants of silver [47] with finite-size corrections according to [35]. The maximum order of the VSHs utilized in the results shown below was $L=8$. The convergence was verified by control calculations with $L=16$. We note that much larger values of $L$ are required for nanospheres in exact contact $(h=0)$ and that the number of the coupled-multipole equations (with complex coefficients) which must be solved to compute the optical responses is equal to $N L(L+2)$.

In Fig. 8 we plot the quantities $S_{i}$ and $\left\langle\left|\mathbf{d}_{i}\right|^{2}\right\rangle /\left(a^{3} E_{0}\right)^{2}$ for three aggregates with fractal dimensions $D \approx 1.70$, $D \approx 1.79$ and $D \approx 1.85$, computed at $\lambda=703 \mathrm{~nm}$. Here $\left\langle\left|\mathbf{d}_{i}\right|^{2}\right\rangle$ is the square of the dipole moment of $i$-th nanosphere averaged over three orthogonal polarizations of the external field. Visual correlation of the two curves is quite apparent. For a more quantitative estimate, we have computed the correlation coefficient $r_{c}\left(S,\left\langle|\mathbf{d}|^{2}\right\rangle\right)$. The dependence of $r_{c}$ on the wavelength is shown in Fig. 9 for three values of fractal dimension and different wavelengths. The maximum degree of correlation is achieved 
for $\lambda=703 \mathrm{~nm}\left(0.69<r_{c}<0.76\right)$. The value of $r_{c}$ decreases monotonously for smaller wavelengths and is in the interval $0.49<r_{c}<0.54$ when $\lambda=505 \mathrm{~nm}$. Note that the correlation coefficient is expected to increase towards unity in the spectral region $\lambda>700 \mathrm{~nm}$. Also, even stronger correlation is expected is a polarizationdependent definition of the local anisotropy factor is used, such as (10) or (11). Validating these hypothesis will be the subject of future work.

\section{SUMMARY}

In this paper we have investigated the statistical correlation between the local geometrical structure and local electromagnetic responses in fractal aggregates of nanoparticles. We have used a realistic aggregation model which allows computer generation of quasi random aggregates of variable fractal dimension in the interval $1.7<D<3.0$. Electromagnetic calculations were carried out using the method of coupled multipoles, i.e., beyond the dipole approximation.

We have found that the local anisotropy factor $S$ introduced in Section $\amalg$ is strongly correlated with the local electromagnetic response. For aggregates built of highquality plasmonic materials, the degree of such correlation tends to increase with the wavelength. The correlation coefficient between the squared dipole moment of a given nanoparticle in an aggregate and a purely geometrical parameter (local anisotropy factor) reaches the value of $\approx 0.75$ for $\lambda=700 \mathrm{~nm}$. We expect that this correlation can become even larger if a properly-defined polarizationdependent local anisotropy factor is used and at larger wavelengths.

The introduced parameter $S$ is a universal geometri- cal characteristic which can be used for analyzing various complicated aggregates and composites without explicit solution of the electromagnetic problem. The discovered strong correlation suggests that, at least in aggregates which are small compared to the wavelength, the large-scale geometry does not play a significant role. Note that in the IR spectral region, subwavelength aggregates can still be built of hundreds or even thousands of nanospheres. The IR spectral region is of special interest because of the very low Ohmic losses in silver and other noble metals. Correspondingly, heterogeneous nanostructures are known to exhibit optical resonances of very high quality. This, in turn, results in giant amplification of local optical responses. The latter phenomenon is currently being actively researched in ordered nanostructures, including self-similar chains of nanospheres (nanolenses) 48] and long chains of similar nanoparticles [49]. Rigorous numerical simulations in random nanoaggregates are still difficult due to the high computational complexity of the associated electromagnetic problem. The introduced parameter $S$ and the discovered correlation of this parameter with local electromagnetic field allows one to make qualitative predictions about the sites where the electromagnetic energy is localized by very simple means, e.g., without solving the electromagnetic problem.

\section{Acknowledgements}

This research was supported by the Russian Foundation for Basic Research, Grant 05-03-32642, and by the Presidium of Russian Academy of Sciences under the Grant 8.1 "Basic Problems of Physics and Chemistry of Nanosize Systems and Nanomaterial".
[1] U. Kreibig and M. Vollmer, Optical Properties of Metal Clusters (Springer-Verlag, Berlin, 1995).

[2] V. M. Shalaev, Phys. Rep. 272(2 \& 3), 61 (1996).

[3] M. I. Stockman, L. N. Pandey, and T. F. George, "Enhanced nonlinear-optical responses of disordered clusters and composites," in Nonlinear Optical Materials (Springer-Verlag, New York, 1998).

[4] V. M. Shalaev, Nonlinear Optics of Random Media: Fractal Composites and Metal Dielectric Films (Springer Verlag, Berlin, 2000).

[5] S. V. Karpov and V. V. Slabko, Optical and Photophysical Properties of Fractal-Structured Metal Sols (Russian Academy of Sciences, Siberian Branch, Novosibirsk, 2003).

[6] V. I. Roldugin, Russian Chemical Review 72(10), 823 (2003), ibid 72(11), 913 (2003).

[7] A. V. Butenko, P. A. Chubakov, Y. E. Danilova, S. V. Karpov, A. K. Popov, S. G. Rautian, V. P. Safonov, V. V. Slabko, V. M. Shalaev, and M. I. Stockman, Z. Phys. D 17, 283 (1990).

[8] M. I. Stockman, V. M. Shalaev, M. Moskovits, R. Botet, and T. F. George, Phys. Rev. B 46(5), 2821 (1992).

[9] F. A. Zhuravlev, N. A. Orlova, V. V. Shelkovnikov, A. I. Plekhanov, S. G. Rautian, and V. P. Safonov, JETP Lett. 56(5), 264 (1992).

[10] V. M. Shalaev, E. Y. Poliakov, and V. A. Markel, Phys. Rev. B 53(5), 2437 (1996).

[11] M. I. Stockman, L. N. Pandey, and T. F. George, Phys. Rev. B 53(5), 2183 (1996).

[12] M. I. Stockman, Phys. Rev. E 56(6), 6494 (1997).

[13] S. V. Karpov, A. K. Popov, S. G. Rautian, V. P. Safonov, V. V. Slabko, V. M. Shalaev, and M. I. Shtokman, JETP Lett. 48(10), 571 (1988).

[14] V. P. Safonov, V. M. Shalaev, V. A. Markel, Y. E. Danilova, N. N. Lepeshkin, W. Kim, S. G. Rautian, and R. L. Armstrong, Phys. Rev. Lett. 80(5), 1102 (1998).

[15] V. A. Markel, V. M. Shalaev, P. Zhang, W. Huynh, L. Tay, T. L. Haslett, and M. Moskovits, Phys. Rev. B 59(16), 10903 (1999).

[16] W. Kim, V. P. Safonov, V. M. Shalaev, and R. L. Armstrong, Phys. Rev. Lett. 82(24), 4811 (1999).

[17] W. D. Bragg, V. A. Markel, W. Kim, K. Banerjee, M. R. 
Young, J. G. Zhu, R. L. Armstrong, V. M. Shalaev, Z. C. Ying, D. Y. E., and V. P. Safonov, J. Opt. Soc. Am. B 18(5), 698 (2001).

[18] J. E. Martin and A. J. Hurd, J. Appl. Cryst. 20, 61 (1987).

[19] M. V. Berry and I. C. Percival, Optica Acta 33(5), 577 (1986).

[20] V. A. Markel, L. S. Muratov, and M. I. Stockman, J. Exp. Theor. Phys. 71(3), 455 (1990).

[21] V. A. Markel, L. S. Muratov, M. I. Stockman, and T. F. George, Phys. Rev. B 43(10), 8183 (1991).

[22] M. I. Stockman, L. N. Pandey, L. S. Muratov, and T. F. George, Phys. Rev. B 51(1), 185 (1995).

[23] M. I. Stockman, T. F. George, and V. M. Shalaev, Phys. Rev. B 44(1), 115 (1991).

[24] V. M. Shalaev, M. I. Stockman, and R. Botet, Physica A 185, 181 (1992).

[25] R. D. Mountain and G. W. Mulholland, Langmuir 4, 1321 (1988).

[26] S. D. Andreev, L. S. Ivlev, E. F. Mikhailov, and A. A. Kiselev, Atmos. Oceanic Opt. 8(5), 355 (1995).

[27] S. D. Andreev and E. F. Mikhailov, Bulletin of the Russian Acad. Sci. 32(6), 743 (1996).

[28] S. V. Karpov, A. L. Bas'ko, A. K. Popov, and V. V. Slabko, Colloid J. 62(6), 699 (2000).

[29] S. V. Perminov, S. G. Rautian, and V. P. Safonov, J. Exp. Theor. Phys. 98(4), 691 (2004).

[30] S. G. Rautian, Opt. Spectrosc. 97(3), 416 (2004).

[31] V. M. Shalaev, R. Botet, and R. Jullien, Phys. Rev. B 44(22), 12216 (1991).

[32] V. M. Shalaev, M. Moskovits, A. A. Golubentsev, and S. John, Physica A 191, 352 (1992).
[33] V. M. Shalaev, R. Botet, and A. V. Butenko, Phys. Rev. B 48(9), 6662 (1993).

[34] V. M. Shalaev and R. Botet, Phys. Rev. B 50(17), 12987 (1994).

[35] V. A. Markel, V. M. Shalaev, E. B. Stechel, W. Kim, and R. L. Armstrong, Phys. Rev. B 53(5), 2425 (1996).

[36] J. E. Sansonetti and J. K. Furdyna, Phys. Rev. B 22(6), 2866 (1980).

[37] J. M. Gerardy and M. Ausloos, Phys. Rev. B 22(10), 4950 (1980).

[38] F. Claro, Phys. Rev. B 25(12), R7875 (1982).

[39] D. W. Mackowski, Appl. Opt. 34(18), 3535 (1995).

[40] Y. E. Danilova, V. A. Markel, and V. P. Safonov, Atmos. Oceanic Opt. 6(11), 821 (1993).

[41] V. A. Markel and V. M. Shalaev, J. Opt. Soc. Am. A 18(5), 1112 (2000).

[42] V. A. Markel, V. N. Pustovit, S. V. Karpov, A. V. Obuschenko, V. S. Gerasimov, and I. L. Isaev, Phys. Rev. B 70(5), 054202 (2004).

[43] D. J. Bergman, Phys. Rep. 43, 377 (1978).

[44] P. Meakin, Phys. Rev. Lett. 51(13), 1119 (1983).

[45] T. A. Witten and L. M. Sander, Phys. Rev. Lett. 47(19), 1400 (1981).

[46] S. M. Heard, F. Griezer, C. G. Barrachough, and J. V. Sandera, J. Colloid Interface Sci. 93(1), 545 (1983).

[47] P. B. Johnson and R. W. Christy, Phys. Rev. B 6(12), 4370 (1972).

[48] K. Li, M. I. Stockman, and D. J. Bergman, Phys. Rev. Lett. 91(22), 227402 (2003).

[49] S. Zou and G. C. Schatz, Chem. Phys. Lett. 403, 62 (2005). 